\title{
Experiencia de 10 años en el manejo del cáncer de pene, Instituto Nacional del Cáncer (1997-2006)*
}

\author{
Penile cancer. A ten years experience
}

\author{
Drs. RODRIGO NEVEU C. ${ }^{1}$, PABLO BÓRQUEZ M. ${ }^{1}$, CRISTIÁN TRUJILLO L. ${ }^{1}$, \\ ROBERTO FERNÁNDEZ R. ${ }^{1}$, MARTÍN BUCHHOLTZ F. ${ }^{1}$
}

${ }^{1}$ Equipo de Cirugía de abdomen y partes blandas, Instituto Nacional del Cáncer, Santiago, Chile

\begin{abstract}
RESUMEN
Introducción: El cáncer epidermoide de pene es una enfermedad infrecuente en occidente, sin embargo tiene alta incidencia en algunos países de Latinoamérica, África y Asia. Se le considera una patología asociada a ciertas costumbres higiénicas y pobres condiciones socioeconómicas. En Chile no hay cifras reales de incidencia del cáncer de pene, pero pareciera ser baja; esto además dificulta la generación de guías y ensayos clínicos para determinar cual es el mejor manejo de los casos que se presentan. El objetivo de este trabajo es presentar la experiencia en el tratamiento del cáncer de pene en el Instituto Nacional del Cáncer entre los años 1997 y 2006. En la actualidad sigue siendo la cirugía el tratamiento de elección para el manejo del tumor primario y los ganglios regionales, asociada a quimioterapia y/o radioterapia. La mayoría de los pacientes consultan en promedio 20 meses después de iniciados los síntomas, lo que determina un estadio muy avanzado (74\% estadios III y IV) al momento del diagnóstico, lo que se traduce en una altísima letalidad y un mal resultado oncológico. La pesquisa de lesiones en estadios precoces, la consulta oportuna, el manejo agresivo de la patología y la concentración del manejo de este cuadro en centros especializados podrían mejorar estos resultados.
\end{abstract}

PALABRAS CLAVE: Cáncer de pene, tratamiento quirúrgico.

\section{SUMMARY}

Background: Penile epidermoid cancer is uncommon and is associated to certain hygienic behaviors and poor economical conditions. There is no information about the incidence of this cancer in Chile, but it is probably low. Aim: To report the experience in the management of penile cancer between 1997 and 2006. Material and methods: Retrospective review of 23 patients aged 26 to 80 years, with a penile cancer. Results: The mean delay between symptom appearance and consultation was 20 months. The cause for consultation was a penile mass in 19, that was ulcerated in 11. Nineteen patients had lymph node involvement and no patient had distant metastases. Therefore, $74 \%$ of patients were in stage II or IV at the moment of consultation. Mean survival was 19, 36, 16 and 8 months for stages I, II, III and IV respectively. Conclusions: In patients with penile cancer, there is a great delay between the onset of symptoms and time of consultation. This results in a high mortality and bad treatment results.

KEY WORDS: Penile cancer, epidermoid carcinoma, mortality.

*Recibido el 31 de Mayo de 2006 y aceptado para publicación el 22 de Octubre de 2007.

Correspondencia: Dr. Rodrigo Neveu C.

Av. Profesor Zañartu 1010, Santiago, Chile

e mail: rodrigoneveu@hotmail.com 


\section{INTRODUCCIÓN}

El cáncer epidermoide de pene (CP) es una neoplasia poco frecuente, aunque es el tipo histológico más frecuente en esta ubicación. En Chile no contamos estadísticas poblacionales respecto a su incidencia, salvo en estudios preliminares efectuados en las ciudades de Antofagasta y Valdivia o en intentos aislados por conocer su epidemiología ${ }^{1}$; en Estados Unidos de Norteamérica representa entre el 0,3 y $0,6 \%$ de los cánceres en hombres y un 2 $\%$ de los cánceres originados en el tracto urogenital masculino ${ }^{2,3}$. En algunos países de África y Asia (como Uganda, China e India) y en algunos sectores de América del Sur como Brasil y Paraguay, constituye un grave problema de salud pública con una incidencia mayor al $10 \%$ de los cánceres masculinos incluso presentando incidencias tan altas como un $20 \%$ de los cánceres en este géne$\mathrm{ro}^{4,5}$. El punto más alto de incidencia de la enfermedad se observa en la sexta década de la vida y se la considera una enfermedad social relacionada con el subdesarrollo y asociada a malas condiciones de higiene local o exposición prolongada a irritantes o cancerígenos desconocidos del esmegma, en individuos no circuncidados, infecciones virales, tabaquismo y radiación ultravioleta 6 . El Virus papiloma Humano (HPV) transmitido sexualmente también ha sido involucrado en el desarrollo de lesiones pre cancerosas y en cáncer de pene propiamente tal, el que asociado a la ausencia de circuncisión parece tener un efecto carcinogénico aún mayor ${ }^{7,8}$.

La tardanza en la consulta y, por tanto lo avanzado del cuadro en el momento de su diagnóstico, han determinado que exista una gran dificultad en su pesquisa precoz y tratamiento exitoso. Por otra parte no existe literatura científica y con un número importante de casos que permitan obtener evidencian de un nivel adecuado para llegar a conclusiones relevantes en relación a su tratamiento

El objetivo de este trabajo es presentar una serie de casos con seguimiento, que resume la experiencia del Instituto Nacional del Cáncer (INC) en el tratamiento de pacientes portadores de cáncer de pene entre los años 1997 y 2006.

\section{MATERIAL Y MÉTODO}

Se presenta una serie de 23 pacientes consecutivos, seleccionados en forma retrospectiva a partir de la base de datos del comité oncológico de nuestra institución. Los pacientes corresponden a hombres portadores de un cáncer de pene ingresados al Instituto Nacional del Cáncer en el período comprendido entre febrero de 1997 y mayo del año
2006. Los pacientes fueron estadificados de acuerdo a la clasificación TNM de la AJCC ${ }^{9}$ (Tabla 1). La evaluación inicial por imágenes incluyó un TAC de abdomen y pelvis y radiografía de tórax en 2 planos para descartar metástasis y demostrar compromiso locorregional. Para el análisis de las distintas variables se utilizó estadística descriptiva y se evaluó la sobrevida por estadio de acuerdo a la curva de Kaplan Meyer. La sobrevida se determinó a partir del momento del diagnóstico con biopsia, hasta el último control o fecha de fallecimiento del paciente. Para el seguimiento se revisó la historia clínica de los pacientes en esta institución, corroborando la fecha de fallecimiento con los datos obtenidos de la oficina de registro civil e identificación, de modo que se logró un $100 \%$ de seguimiento de nuestros enfermos.

Tabla 1

AGRUPACIÓN POR ESTADIOS DEL AJCC

\begin{tabular}{|c|c|c|c|}
\hline ESTADIO & $T$ & $N$ & $M$ \\
\hline \multirow[t]{2}{*}{0} & is & 0 & 0 \\
\hline & a & 0 & 0 \\
\hline ESTADIO I & 1 & 0 & 0 \\
\hline \multirow[t]{3}{*}{ ESTADIO ॥ } & 1 & 0 & 0 \\
\hline & 2 & 0 & 2 \\
\hline & 2 & 1 & 0 \\
\hline \multirow[t]{5}{*}{ ESTADIO III } & 1 & 2 & 0 \\
\hline & 2 & 2 & 0 \\
\hline & 3 & 0 & 0 \\
\hline & 3 & 1 & 0 \\
\hline & 3 & 2 & 0 \\
\hline \multirow[t]{3}{*}{ ESTADIO IV } & 4 & Cualquier N & 0 \\
\hline & Cualquier T & 3 & 0 \\
\hline & Cualquier $\mathrm{T}$ & Cualquier $\mathrm{N}$ & 1 \\
\hline
\end{tabular}

Tumor primario $(\mathrm{T})$

TX: No puede evaluarse el tumor primario

TO: No hay evidencia de tumor primario

Tis: Carcinoma in situ

Ta: Carcinoma verrugoso no invasor

T1: Tumor invade tejido conectivo subepitelial

T2: Tumor invade cuerpo esponjoso o cavernoso

T3: Tumor invade uretra o próstata

T4: Tumor invade otras estructuras adyacentes

Ganglios linfáticos regionales (N)

NX: No pueden evaluarse los ganglios linfáticos regionales

No: No hay metástasis a los ganglios linfáticos regionales

N1: Metástasis en un solo ganglio linfático superficial inguinal

N2: Metástasis en ganglios linfáticos inguinales superficiales múltiples o bilaterales

N3: Metástasis en ganglio(s) linfático(s) profundo(s) inguinal(es) o pélvico(s) unilateral(es) o bilateral(es)

Metástasis a distancia (M)

MX: No puede evaluarse la metástasis a distancia

M0: No hay metástasis a distancia

M1: Metástasis a distancia 
Los distintos procedimientos a los que fueron sometidos estos pacientes comprendieron biopsia, circuncisión, penectomía parcial, emasculación, una disección inguinal uni o bilateral y algunos enfermos además recibieron radio y/o quimioterapia. En aquellos pacientes que recibieron quimioterapia se usaron esquemas en base a Mitomicina $(10 \mathrm{mg} / \mathrm{m} 2 \mathrm{el}$ día uno) asociado a 5 Fluoracilo $(1000 \mathrm{mg} / \mathrm{m} 2$ en infusión continua los días 1 a 4 ó 5), Cisplatino (100 $\mathrm{mg} / \mathrm{m} 2$ día 1) asociado a 5 Fluoracilo. La radioterapia curativa se realizó con dosis de entre 36 y 66 Gy, con intención paliativa se entregaron dosis para completar 30 Gy en 10 fracciones.

\section{RESULTADOS}

Esta serie de 23 pacientes tenía una edad promedio de 68 años (rango 26 a 80 años) al momento de la primera consulta, todos se encontraban en relativas buenas condiciones generales al momento de ingresar a nuestro servicio, con un performance status según ECOG de 2 o menor, lo que permitió ofrecer algún tipo de tratamiento a la mayoría de ellos. Sin embargo cabe destacar que el tiempo promedio de consultas fue 19,6 meses después de la aparición de los primeros síntomas. Respecto de la presentación clínica, 19 pacientes consultaron por presentar una masa peneana, en 11 pacientes ulceradas, que en tres casos eran dolorosas. En cuatro casos existe presencia de fimosis. La ubicación del tumor primario se aprecia en la Tabla 2.

La Tabla 3 muestra la clasificación de los pacientes según TNM. De los 19 pacientes con compromiso ganglionar, 12 presentaba compromiso bilateral. Ningún paciente tenía metástasis a dis-

Tabla 2

\section{UBICACIÓN DEL TUMOR PRIMARIO}

\begin{tabular}{lr}
\hline Glande & 13 \\
Prepucio & 6 \\
Surco balano prepucial & 2 \\
Uretra & 2 \\
Total & 23 \\
\hline
\end{tabular}

Tabla 3

CLASIFICACIÓN DE LOS PACIENTES SEGÚN ESTADIO.

\begin{tabular}{lcc}
\hline Estadio & $\mathrm{N}$ & $\%$ \\
I & 1 & 4.3 \\
II & 5 & 21.7 \\
III & 13 & 56.5 \\
IV & 4 & 17.4 \\
Total & 23 & 100 \\
\hline
\end{tabular}

Tabla 4

\section{PROCEDIMIENTOS EFECTUADOS A 23 PACIENTES CON CÁNCER DE PENE}

\begin{tabular}{lr}
\hline Procedimiento & $N$ \\
\hline Biopsia & 19 \\
Circuncisión & 3 \\
Disección Inguinal & 7 \\
Disección Inguinal Bilateral & 3 \\
Emasculación & 5 \\
Penectomia Parcial & 17 \\
Radio/Quimioterapia & 11 \\
Radioterapia Paliativa & 5 \\
\hline
\end{tabular}

La mayor parte de los pacientes fueron sometidos a más de un procedimiento

tancia al momento de la consulta, los pacientes en estadio IV fueron diagnosticados a raíz de un compromiso linfático masivo (N3). Como resultado de la estadificación, observamos que la gran mayoría de los pacientes consultaron en estadios avanzados (III y IV). El grado histológico de malignidad fue de G1: $26,1 \%$, G2: $39,1 \%$ y G3:34,8 \%.

17 de los 23 pacientes (73,9\%) habían sido intervenidos con algún procedimiento mayor antes de su llegada al hospital. Los procedimientos a los que fueron sometidos se observan en la Tabla 4. La sobrevida promedio fue de 19 meses para el estadio I, 36 meses para el estadio II, 15,6 meses para el estadio III, y 7,5 meses para el estadio IV; la sobrevida actuarial con curvas de Kaplan Meyer según estadio clínico puede verse en la Figura 1; sólo tres pacientes permanecen vivos al final del seguimiento de esta casuística.

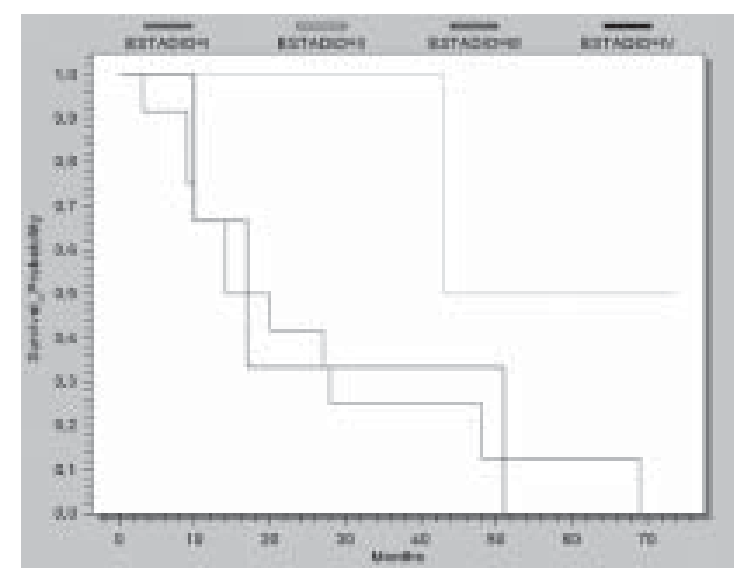

Figura 1. Sobrevida de 23 pacientes con Cáncer de Pene según Estadio (TNM). 


\section{DISCUSIÓN}

A pesar de lo avanzado de la enfermedad de nuestros casos, es llamativo que más de un $80 \%$ de ellos llegaron en condiciones adecuadas de tratamiento (ECOG 1 y 2), lo que permitió realizar algún tipo de terapia con cirugía y quimioterapia y/ o radioterapia. Así como se pudo tratar a un gran porcentaje de ellos a su llegada a nuestra institución, es visible que los resultados finales de esta serie reflejan un resultado oncológico muy lejos de lo deseado. Creemos que la alta mortalidad de esta casuística se debe a dos factores principales: el primero es que nuestros pacientes ya sea por negligencia, temor o ignorancia consultaron en promedio luego de aproximadamente 20 meses desde el inicio de los síntomas, cuándo es un hecho conocido que un enfermo con cáncer de pene dejado evolucionar sin tratamiento usualmente fallece en un lapso de dos a tres años, de modo tal que nuestros casos al momento de la consulta ya presentaban pronóstico ominoso; así, pese a nuestros esfuerzos se comportaron como una población no tratada dejada a una evolución natural de la enfermedad $^{6}$. El segundo factor que nos parece primordial en estos resultados es el hecho de que un altísimo porcentaje de ellos fueron tratados previamente, no siempre con un criterio oncológico adecuado, requiriendo muchos extensas cirugías de rescate.

En Chile no disponemos de datos reales de incidencia de enfermedad salvo en zonas que cuentan con registros poblacionales de tumores (Concepción, Antofagasta y Valdivia) y en algunos lugares donde se han realizado algunos esfuerzos puntuales por conocer la epidemiología de los tumores urológicos ${ }^{1}$. Tanto en estos trabajos como en nuestra experiencia previa ${ }^{8}$, hemos podido constatar la alta mortalidad de este cuadro debido al extenso compromiso locorregional y a lo prolongado del tiempo de evolución antes de la consulta al médico, hechos que coinciden con la literatura internacional ${ }^{11-13}$. Asimismo la forma de presentación clínica y la ubicación del tumor primario nuestra serie no difiere de otras publicadas ${ }^{12}$.

Otro factor pronóstico importante es el grado de diseminación a linfonodos regionales ${ }^{5,14-17}$. Sabemos que la sobrevida de los pacientes con linfonodos positivos se reduce notoriamente (en promedio rara vez supera los dos años). También sabemos que la aparición de las adenopatías metástasicas demora unos seis meses en ser evidente clínicamente (con un rango de 2 a 12 meses) ${ }^{13}$. Estos factores son los que explican en gran medida el pobre resultado oncológico de nuestra serie, pues casi el $90 \%$ de nuestros pacientes presentaban linfadenopatías al momento de ingresar a nuestro servicio. A pesar de que evaluando nuestra experiencia anterior ${ }^{10}$, en la década del 90 cambiamos nuestra postura siendo mucho más agresivos y agregando radio quimioterapia a las etapas avanzadas, no conseguimos los resultados esperados, persistiendo una alta mortalidad en las etapas avanzadas.

Las metástasis a distancia son inusuales en ausencia enfermedad regional inguinal, y ocurren en menos de un $10 \%$ de los pacientes, normalmente a pulmón, hígado, huesos y cerebro; sin embargo y pese a la extensión del compromiso regional, ninguno de nuestros enfermos la presentaba al momento de consultar.

Los casos portadores de lesiones pequeñas que permiten una penectomía parcial con bordes oncológicos libres de tumor y sin compromiso linfático tienen una sobrevida a 5 años de aproximadamente $80 \%$. Este hecho nos permite remarcar dos conceptos: En primer lugar la necesidad de educar respecto de la importancia de la consulta precoz ante la aparición de estas u otras lesiones. Debido a que es un tumor de crecimiento lento podría ser diagnosticado en etapas precoces, por tanto no cabe duda de que los avances en el pronóstico de este cuadro deben ir dirigidos a la detección en etapas de lesión preinvasora, o bien cuando el cáncer de pene se encuentra en estado invasor de menos de $2 \mathrm{~cm}$. En segundo lugar, en este grupo el tratamiento precoz ha dado buenos resultados en lo que la conservación de órgano y sobrevida se refiere, y a la utilización del linfonodo centinela como una medida de detectar precozmente la eventual existencia de metástasis linfáticas loco-regionales. La literatura ha demostrado que este procedimiento es factible y además podría permitir el eventual tratamiento precoz de la diseminación con mejoría de los resultados a largo plazo $5,16,25$.

El cáncer de pene es una patología infrecuente en Chile, generalmente los pacientes consultan en etapas tardías, y con diseminación locorregional, factores que se asocian a un pronóstico ominoso. Estimamos que el manejo de esta enfermedad se debe enfocar a la educación para la detección precoz con el objetivo de intentar obtener un control más efectivo de la enfermedad locorregional diseminada.

\section{REFERENCIAS}

1. Navarro M, Montes J, Tagle. R. Epidemiología de los cánceres urológicos en la Tercera Región de Atacama. Rev Chil Urol 2004; 69: 230-236. 
2. Carver B, Mata J, Venable D, Eastham J. Squamous cell carcinoma of the penis: a retrospective review of forty-five patients in Northwest Louisiana. South Med J 2002; 44: 822-825.

3. Micali G, Nasca MR, Innocenzi D, Schwartz RA. Penile cancer. J Am Acad Dermatol 2006; 54: 369591.

4. Campos RS, Lopes A, Guimaraes GC, Carvalho AL, Soares FA. E-cadherin, MMP-2, and MMP-9 as prognostic markers in penile cancer: analysis of 125 patients. Urology 2006; 67: 797-802.

5. Pompeo AC. Extended lymphadenectomy in penile cancer. Can J Urol 2005; 12 Suppl 1: 30-36.

6. Misra S, Chaturvedi A, Misra N. Penile carcinoma: a challenge for the developing world. Lancet Oncol 2004; 55: 240-247.

7. Castellsague X, Bosh FX, Munoz F, Meijer CJ, Shah $\mathrm{KV}$, De Sanjose $S$ et al. Male circumcision, penile human papilloma virus infection, and cervical cancer in female. N Engl J Med. 2002; 346: 1105-1112.

8. Watson R. Human Papilloma Virus: Confronting the epidemic-A urologist's perspective. Rev Urol 2005; 135: 144.

9. Penis cancer. En Greene F, Page D, Fleming I, Fritz A, Blach Ch, Haller D, et als, eds. Cancer Staging Manual AJCC. Springer, 2002.

10. Trujillo C, Ristori L, Martín SS, Arraztoa J. Cancer de pene. Complicaciones del tratamiento quirúrgico. Rev Chil Cir 1991; 43: 307-311.

11. Das S. Penile amputations for the management of primary carcinoma of the penis. Urol Clin North Am 1992; 19: 277-282.

12. Burgers JK, Badalament RA, Drago JR. Penile cancer. Clinical presentation, diagnosis, and staging. Urol Clin North Am 1992; 19: 247-256.

13. Bengió R, Ariel F, Lenkiewicz I, Villarreal C, Orellana $\mathrm{S}$, Olmedo. L. Cáncer de pene, revisión de la casuística. Junio de 1995 a junio de 2002. Rev Chil Urol 2003; 68: 156-161.

14. Perdona S, Autorino R, Gallo L, Di Lorenzo G, Cascini GL, Lastoria F, et al. Role of dynamic sentinel node biopsy in penile cancer: our experience. J Surg Oncol 2006; 93: 181-185.

15. Borchers H, Jakse G. Lymphadenectomy for penile cancer. Diagnostic and prognostic significance as well as therapeutic benefit. Urologe A 2005; 44: 657661.

16. Siow WY, Cheng C. Penile cancer: current challenges. Can J Urol 2005; 12 Suppl 1: 18-23; discussion 97-8.

17. Stancik I, HoltI W. Penile cancer: review of the recent literature. Curr Opin Urol 2003; 13: 467-472.

18. Izawa J, Kedar D, Wong F, Pettaway CA. Sentinel lymph node biopsy in penile cancer: evolution and insights. Can J Urol 2005; 12 Suppl 1: 24-29.

19. Tanis PJ, Lont AP, Meinhardt W, Olmos RA, Nieweg OE, Horenblas S. Dynamic sentinel node biopsy for penile cancer: reliability of a staging technique. J Urol 2002; 168: 76-80.

20. Akduman B, Fleshner NE, Ehrlich L, Klotz L. Early experience in intermediate-risk penile cancer with sentinel node identification using the gamma probe. Urology 2001; 58: 65-68.

21. Wawroschek F, Vogt H, Bachter D, Weckermann D, Hamm M, Harzmann R. First experience with gamma probe guided sentinel lymph node surgery in penile cancer. Urol Res 2000; 28: 246-249.

22. Pettaway CA, Pisters LL, Dinney CP, Jularbal F, Swanson DA, von Eschenbach AC et al. Sentinel lymph node dissection for penile carcinoma: the $M$. D. Anderson Cancer Center experience. J Urol 1995; 154: 1999-2003.

23. Srinivas $V$, Joshi A, Agarwal B, Mundhada $U$, Shah A, Phadke AG. Penile cancer-the sentinel lymph node controversy. Urol Int 1991; 47: 108-109.

24. Fowler JE, Jr. Sentinel lymph node biopsy for staging penile cancer. Urology 1984; 23: 352-353.

25. Evaluación de ganglios regionales en pacientes con carcinoma de pene mediante dos técnicas nucleares: resultados preliminares. Universidad de Chile, 2006. (Accessed 12/28/2006 2006, at http:// www2.alasbimnjournal.cl/alasbimn/CDA/sec_a/ 